\title{
LA POSSIBILITÉ D'ÉVITER DES OVIPOSITIONS IRRÉGULIÈRES GRÂCE AU NANISME LIÉ AU SEXE.
}

\author{
A.R. KUIT
}

Institut Het Spelderholt

Beekbergen (Hollande)

\begin{abstract}
Des ovipositions irrégulières sont plus fréquentes chez les mères de type "chair " que chez les pondeuses. Deux œufs pondus le même jour rendent compte de 0,8 à 6,2 p. 100 des jaunes pour les types "broiler ", comparés à 0,1 à 2,0 p. 100 pour des poulettes de type "pondeuse ", dans différentes lignées, au début de la période de ponte (Jaap et Muir, 1968).

Des recherches de Van Middel koop à l'Institut de Recherches avicoles Spelderholt indiquent que la majorité des œufs pondus à moins de 20 heures d'intervalle présente une anomalie particulière due à la présence de deux œufs dans la glande coquillière. Ces cufs ont dans l'ensemble une très mauvaise éclosion.

La possibilité d'un effet régulateur du gène de nanisme lié au sexe sur l'oviposition est fiscutée.
\end{abstract}

\section{COMPORTEMENT DES FEMELLES NAINES EN TESTAGE}

A IA STATION DE PLOUFRAGAN

SPÉCIALEMENT VIS-A-VIS DE LA MALADIE DE MAREK

\section{MEURIER}

\author{
Station Expérimentale d'Aviculture \\ 22 - Ploutragan (France)
}

Au cours des 3 dernières années, il nous a été donné d’étudier le comportement et les performances de femelles $d w$ dans le cadre des tests comparatifs des variétés de reproducteurs de type chair présentes sur le marché français.

Dans le test 1968-1969 la $J V$ 15, vedette INRA lourde, était présente ; la seule observation intéressante que nous ayons pu faire concerne son comportement en présence d'un pourcentage de coqs considérés comme normal pour les pondeuses ordinaires à savoir $10 \mathrm{p}$. 100. Les parquets de ce test abritaient 30 poules et, alors que les pondeuses normales toléraient facilement 4 coqs, les naines n'en toléraient que 2. La mortalité moyenne pendant les 24 premières semaines s'étant élevée à 6,23 p. 100 pour l'ensemble des lots et à 2,5 p. 100 pour la JV 15 , et, pendant la ponte à 6,2 p. 100 pour l'ensemble des lots et à 2,5 p. 100 pour la JV 15 , on ne peut dire que dans des conditions sanitaires satisfaisantes la JV 15 ait eu une viabilité supérieure à celle des poules normales.

Dans le test 1969-1970, la $J V$ 15, vedette INRA lourde, à plumage blanc, est à nouveau présente, ainsi que, pour la première fois, la $J A 57$ ou vedette INRA légère, à plumage rouge. Les poids respectifs de la vedette légère, de la vedette lourde et des souches normales s'élevaient à 24 semaines à 1.900 g., $2.100 \mathrm{~g}$. et $3.000 \mathrm{~g}$. environ et à l'âge d'un an à $2.200 \mathrm{~g} .2 .600 \mathrm{~g}$. et 3.650 g. environ.

Du point de vue sanitaire, la période d'élevage des futurs reproducteurs fut marquée par l'apparition au cours de la $10^{\mathrm{e}}$ semaine de la forme aiguë de la Maladie de Marek qui provoqua la perte par mortalité de 33 p. 100 des femelles et de 18 p. 100 des mâles de l'ensemble du troupeau jusqu'à 24 semaines, puis de 16 p. 100 des femelles restantes de 24 semaines à 1 an. Or jusqu'à 
24 semaines, la mortalité totale đes vedettes légères, des vedettes lourdes et des poulettes normales s'est élevée respectivement à 9,4 p. 100,35 p. 100 et 43 à 57 p. 100 et la mortalité par Marek à 3 p. 100,26 p. 100 et 38 à 52 p. 100 . De 24 à 52 semaines, la mortalité totale s'est élevée respectivement à 4,2 p. 100,30 p. 100 et 30 à 50 p. 100 et la mortalité par Marek à 1,7 p. 100, 17 p. 100 et 14 à $27 \mathrm{p}$. 100. La vedette légère apparait donc non seulement comme très résistante à la Maladie de Marek en période d'élevage et de ponte, mais également comme douée d'une viabilité générale très supérieure à la moyenne des autres souches de type chair. La vedette lourde quant à elle semble, en période d'élevage, moins sensible à la Maladie de Marek sans qu'on puisse donner ce résultat comme significatif ; par contre, en période de ponte elle se révèle tout à fait comparable aux variétés de pondeuses normales.

Il convient de préciser que la dissémination de l'agent de la maladie à l'ensemble des lots a eu lieu pendant les 8 premières semaines d'élevage et qu'elle a été favorisée par le fait que les différents parquets n'étaient séparés que par une simple cloison grillagée, le virus se transmettant essentiellement par voie aérienne.

L'hypothèse d'une résistance génétique à la Maladie de Marek due à la présence du gène $d w$ semblait donc parfaitement valable, la solirlité de cette résistance pouvant être liée à l'association de ce gène avec un gène $s$ ou un poids moins élevé.

Dans le test actuellement en cours (1970-1971), nous retrouvons la $J V 15$ et la $J A 5 z$ dont les poids de 1 jour à 24 semaines sont identiques à ceux du test précédent. Une fois encore, la Maladie de Marek est apparue sur ce troupeau, mais plus tardivement et avec une intensité moindre : le $1^{\text {er }}$ cas diagnostiqué le fut là 15 semaines et sur une poulette $J A 57$ (vedette rouge légère) ; cependant, alors que pour l'ensemble du troupeau le taux de mortalité par Marek s'élève à 5 p. 100 à 24 semaines et à 8,5 p. 100 à 40 semaines, celui de la $J A 57$ n'atteint que 1,2 p. 100 à 24 semaines et 1,7 p. 100 à 40 semaines. L'hypothèse d'une résistance génétique particulière de la $J A 57$ à la Maladie de Marek est donc confirmée ; le premier cas de maladie ayant été diagnostiqué sur cette souche, on est certain qu'elle a bien été contaminée comme les autres. Par contre, la mortalité par Marek des $J V 15$ (vedeltes blanches lourdes) a atteint en moyenne 9 p. 100 à 24 semaines et 15 p. 100 à 40 semaines, détruisant ainsi l'hypothèse que par sa seule présence le gène $d w$ confère une résistance particulière à la Maladie de Marek.

Une étude de la résistance génétique à la Maladie de Marek après infection expérimentale de poussins possédant ou non le gène $d w$ est actuellement effectuée en collaboration avec MérAT.

\title{
QUEIQUES PARTICULARITÉS NUTRITIONNELLES ET PHYSIOLOGIQUES DU POUSSIN $d w$
}

\author{
J. GUILLAUME
}

Station de Recherches avicoles, I.N.R.A., 37 - Nouzilly (France)

On possède très peu d'informations sur les besoins nutritionnels du poussin dw en croissance comparé au poussin normal. Ayant trouvé dans une expérience précédente que le poussin $d w$ utilisait l'énergie de façon plus efficace et les protéines moins efficacement, nous avons entrepris de déterminer s'il existe ou non une interaction entre le taux protidique et le génotype dans trois expériences différentes de courte durée. Tous les animaux étaient des poussins pedigree ou semipedigree et provenaient d'un croisement $D w d w \times \mathrm{x} d w-$. Par suite, nous avions 50 p. 100 de poussins normaux et 50 p. 100 de nains de chaque sexe. Les phénotypes étaient déterminés à la fin des expériences, à 12 semaines d’âge.

Dans la première expérience, tous les oiseaux étaient nourris par la méthode de «l'alimentation séparée ". Ils recevaient une quantité journalière limitée d'un aliment riche en protéines et, dans une autre mangeoire, un aliment sans protéines, ad libitum. Trois quantités différentes d'aliments 